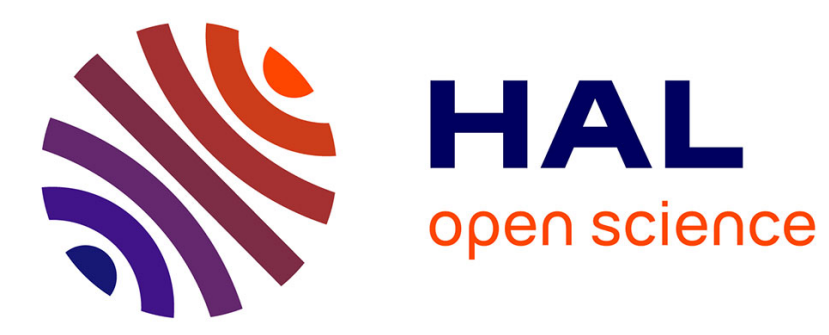

\title{
Mind the stimulation site: Enhancing and diminishing sentence comprehension with anodal tDCS
}

Alessandra Vergallito, Erica Varoli, Beatrice Giustolisi, Carlo Cecchetto, Lilia del Mauro, Leonor Romero Lauro

\section{- To cite this version:}

Alessandra Vergallito, Erica Varoli, Beatrice Giustolisi, Carlo Cecchetto, Lilia del Mauro, et al.. Mind the stimulation site: Enhancing and diminishing sentence comprehension with anodal tDCS. Brain and Language, 2020, 204, pp.104757. 10.1016/j.bandl.2020.104757 . hal-03049254

\section{HAL Id: hal-03049254 \\ https://hal.science/hal-03049254}

Submitted on 20 Jul 2021

HAL is a multi-disciplinary open access archive for the deposit and dissemination of scientific research documents, whether they are published or not. The documents may come from teaching and research institutions in France or abroad, or from public or private research centers.
L'archive ouverte pluridisciplinaire HAL, est destinée au dépôt et à la diffusion de documents scientifiques de niveau recherche, publiés ou non, émanant des établissements d'enseignement et de recherche français ou étrangers, des laboratoires publics ou privés. 
This a pre-final version 
Mind the stimulation site: enhancing and diminishing sentence comprehension with anodal tDCS

Alessandra Vergallito ${ }^{1,2+}$, Erica Varoli ${ }^{2,3,4+}$, Beatrice Giustolisi ${ }^{1}$, Carlo Cecchetto ${ }^{1,5}$, Lilia Del Mauro $^{1}$ \& Leonor J. Romero Lauro* ${ }^{1,2}$

${ }^{1}$ Department of Psychology, University of Milano-Bicocca, Italy

${ }^{2}$ Milan Center for Neuroscience, Neuromi, Italy

${ }^{3}$ Ph.D. Program in Neuroscience, Department of Medicine and Surgery, University Milano-Bicocca, Monza, Italy

${ }^{4}$ Psychology Service, Department of Diagnostic and Therapeutic Services, Mediterranean Institute for Transplantation and Advanced Specialized Therapies (IRCCS-ISMETT), Palermo, Italy

${ }^{5}$ Université de Paris 8 \& CNRS - UMR 7023 Structures Formelles du Langage, Paris, France

$+\mathrm{AV}$ and EV equally contributed to the manuscript

Word count $=4838$

*Corresponding author:

leonor.romero1@unimib.it,

Department of Psychology, University of Milano Bicocca,

Piazza Ateneo Nuovo, 1, 20126 Milano, Italy. 


\begin{abstract}
In a previous sham-controlled study, we showed the feasibility of increasing language comprehension in healthy participants by applying anodal transcranial direct current stimulation (atDCS) over the left inferior frontal gyrus (LIFG). In the present work, we present a follow-up experiment targeting with atDCS the left inferior parietal cortex (LIPC) while participants performed the same comprehension task used in our previous experiment. Both neural sites are crucial hubs of Baddeley's model of verbal short-term memory (vSTM).

AtDCS over LIPC decreased accuracy as compared to sham and LIFG stimulation, suggesting the involvement of these brain regions in sentence comprehension. Crucially, our results highlighted that applying tDCS over different hubs of the same neural network can lead to opposite behavioral results, with relevant implications from a clinical perspective.
\end{abstract}

Keywords

Sentence comprehension, tDCS, verbal short-term memory, language processing, syntactic processing 


\section{Introduction}

Transcranial direct current stimulation (tDCS) is broadly used in experimental research to modulate behavioral performance in healthy participants and as a promising coadjutant treatment from a clinical-rehabilitation perspective. To reinforce its application with neurological patients, more evidence is required to find which protocols are more suitable in inducing behavioral benefits. With this objective in mind, in a previous study (Giustolisi et al., 2018) and in the present experiment, we explored the feasibility to modulate sentence comprehension by stimulating with tDCS two cortical hubs, namely the left inferior frontal gyrus (LIFG or left BA44) and the left inferior parietal cortex (LIPC or left BA40). These two sites were chosen because previous evidence from neuropsychological, neuroimaging and brain stimulation studies (e.g. Vallar and Papagno, 2002; Paulesu et al., 1993; Romero Lauro et al., 2006; see Shallice and Papagno, 2019 for a recent review) indicated these regions as the neural correlates of the specific components of verbal short-term memory (vSTM), namely the rehearsal and phonological store, according the phonological loop model proposed by Baddeley \& Hitch (1974). The rationale behind LIFG and LIPC stimulation was to improve sentence comprehension by enhancing vSTM capacity. Actually, there is a longstanding and still unsolved debate on the relationship between vSTM and language processing. Caplan and Waters (1999), for instance, claim for a role of a separate STM system specific to syntax, with working memory - and more specifically - the central executive component playing a role only in a post-interpretative stage. According to another view, no link is traceable between STM and sentence comprehension (Butterworth, Campbell, \& Howard, 1986) with the coupling of deficits in STM and language tasks being due just to comorbidities. Other authors argue for the existence of a specific semantic component of STM, separated from vSTM, crucial for sentence comprehension (Martin \& Romani, 1994; Martin \& He, 2004). However, in the last ten years our research group collected converging evidence indicating that LIFG and LIPC are indeed causally involved in vSTM tasks (Romero Lauro et 
al., 2006) and that vSTM plays a role in the comprehension of syntactically complex sentences (Romero Lauro et al., 2010; Papagno \& Cecchetto, 2019). For instance, repetitive transcranial magnetic stimulation (rTMS) over LIFG and LIPC disrupted performance on both digit span and sentence comprehension task (Romero Lauro et al., 2010). Therefore, it seems legitimate to explore the hypothesis that an excitability enhancement of LIFG or LIPC would have boosted vSTM ability, which in turn would have improved sentence comprehension, particularly in the case of syntactically complex sentences (Friederici, 2011; Romero Lauro et al., 2010; Papagno and Cecchetto, 2019; Thothathiri et al., 2012; Wilson et al., 2016).

Specifically, in our previous experiment we showed for the first time that the application of anodal tDCS over LIFG can increase language comprehension in healthy participants. Our results showed an overall increase in participant's accuracy during anodal tDCS as compared to a sham/placebo condition, not modulated by syntactic complexity.

To complement this result, in the current study we decided to stimulate LIPC. We then recruited 22 healthy participants who performed the same sentence-to-picture matching task as the previous studies (Giustolisi et al., 2018; Romero Lauro et al., 2010) while anodal tDCS was applied over LIPC. The first reason to choose LIPC as a stimulation site is that anodal tDCS over LIPC, by fostering the phonological store activity, might enhance sentence comprehension (Papagno et al., 2017; Paulesu et al., 1993; Romero Lauro et al., 2010; Pisoni et al., 2019). A second reason is that recent studies propose that LIPC might be part of a wide network directly involved in syntactic processing (Thothathiri et al., 2012; Wilson et al., 2016; see Friederici, 2011 for a review). As our main goal is exploring which type of tDCS protocols can boost language comprehension in healthy subjects (and ultimately in patients), LIPC seemed a reasonable candidate.

\section{Methods}

2.1 Participants 
Data from sixty-six Italian-native students (18 males, Mage $=22.3 \pm 2.2)$ were analysed in the study. 44 took part in a previous experiment (Giustolisi et al., 2018) in which they were randomly assigned to anodal tDCS over the LIFG (males $=11$, Mage $=22.5, \pm 1.77$ ) vs sham $/$ placebo condition $($ males $=1$, Mage $=22.7, \pm 2.39$ ). Data from 22 participants were collected ex-novo to this specific experiment, and they were all assigned to anodal tDCS over the left inferior parietal lobule $($ males $=6$, Mage $=21.8, \pm 2.16)($ LIPC). The three groups differed in terms of gender $\left(\chi_{(2)}^{2}=8.3, p=.016\right)$, but not age $\left(\chi_{(2)}^{2}=0.2, p=.989\right)$.

Both the experiments were performed in single-blind: indeed, participants were naïve to the experimental procedure, and they were not informed about the purpose of the study until the debriefing. However, experimenter interaction with participants were limited: indeed, after tDCS electrodes montage participants wore earphones until the end of the experiment.

All participants completed the Adult Safety Screening Questionnaire (Keel et al., 2001) and gave informed written consent prior to study procedures. Students with any contraindication to tDCS administration did not take part in the experiment (Rossi et al., 2009). Participants were right-handed according to the Edinburgh Handedness Inventory (Oldfield, 1971) (mean laterality coefficient $=.82, \mathrm{SD}=.14)$. The study was approved by the Ethical Committee of the University of Milano - Bicocca.

\section{2 tDCS parameters}

TDCS was delivered using a BrainSTIM stimulator (EMS) through two electrodes: the anode, which measured $3 \times 3 \mathrm{~cm}$, and the cathode, with a size of $7 \times 5 \mathrm{~cm}$. In the anodal and sham LIFG conditions, the anode was placed over F5, whereas in the LIPC one over P3 according to EEG 10-20 system (e.g. Herwig et al., 2003). In both montages, the cathode was applied over right supraorbital area. A constant current with an intensity of $0.75 \mathrm{~mA}$ was applied for $30 \mathrm{~min}$ in LIFC and LIPC anodal stimulation, with $10 \mathrm{~s}$ of fade-in/fade-out period during which current ramped on 
and off. In the sham tDCS, stimulator turned off automatically after $30 \mathrm{~s}$, a procedure typically used to blind participants from their assigned condition (e.g. Gandiga et al., 2006). Figure 1 represents the estimated electric fields induced by tDCS in the LIFG and LIPC conditions computed with Comets software (http://www.COMETStool.com, Jung et al., 2013). (see Figure 1 for the estimate electric field induced by the stimulations and computed by using Comets software).

A

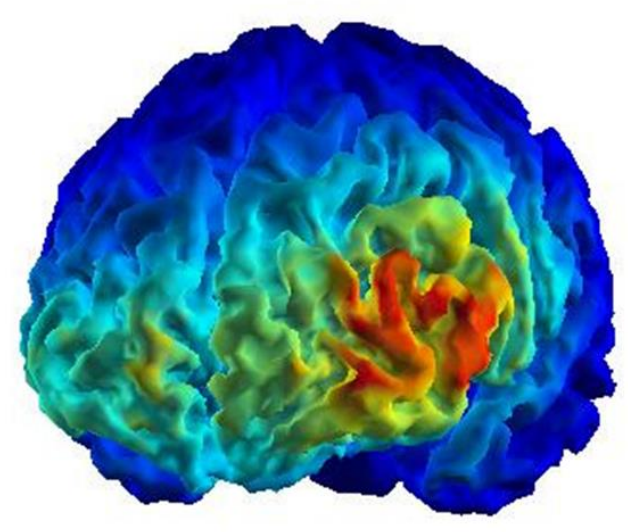

C

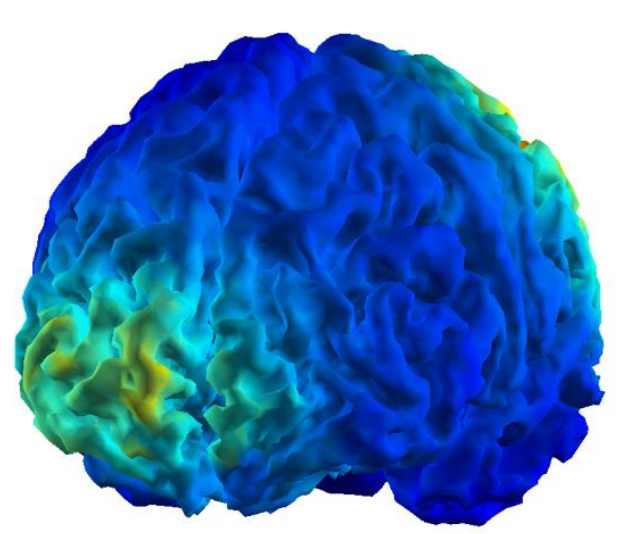

B

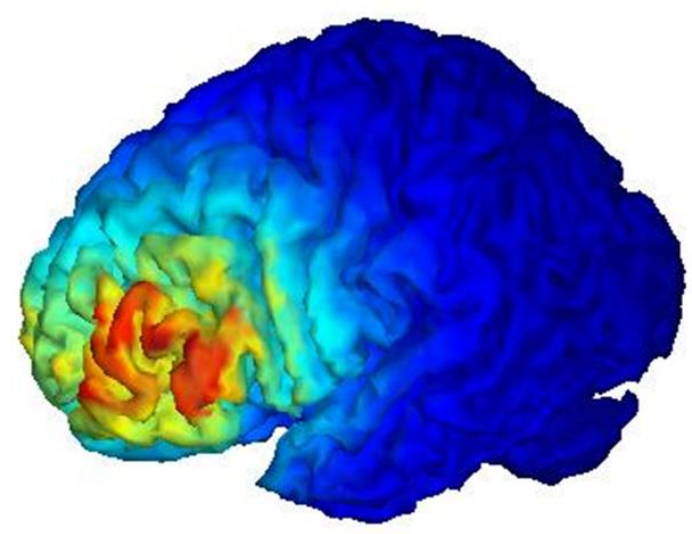

D

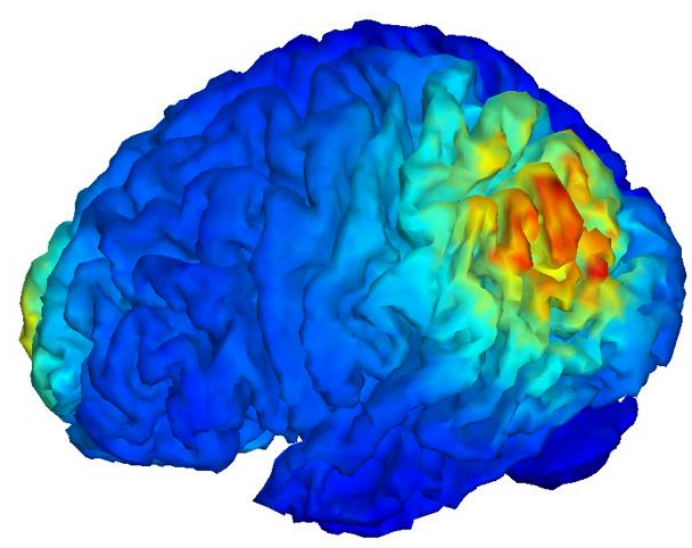

Figure 1. The figure represents the estimated electric field induced by tDCS. Specifically, at the top of the figure Panel A shows the electric field induced by the cathode (reference electrode) over the right supraorbital region, while Panel B displays the anode (target electrode) positioned over the LIFG. At the bottom of the figure, electrical distribution is computed for the cathode (supraorbital region, Panel C) with the anode placed over the LIPC (Panel D). 


\subsection{Linguistic and control tasks}

Linguistic task consisted in a modified version of the standardised Italian battery “COMPRENDO" (Cecchetto et al., 2012). 90 pre-recorded sentences with different syntactic complexity were acoustically presented, each one paired with two pictures appearing $1000 \mathrm{~ms}$ before the sentence end. Specifically, sentence sample included 36 fillers (active and passive simple sentences), plus 54 targets comprising coordinate (Coord) and relative clause in centerembedded (Rel_CE) or right peripheral (Rel_RP) position. Participants were asked to choose the picture which correctly represented sentence meaning, while the wrong one depicted the same situation but with character playing opposite roles. Control task consisted in a short-term memory visual pattern task (VPT), prepared using checkerboards with black and white squares. In the task a screen-centred checkerboard was presented, followed by two alternative stimuli, one equal to the first checkerboard, one differing for the position of one square. Participants were asked to choose the identical checkerboard. VPT comprises 18 trials and individual visual span (determined by checkerboard size) were set before tDCS delivering at $80 \%$ of accuracy.

After giving their written informed consent, individual span was established. Then, the experimenter proceeded with tDCS electrodes montage and participants were asked to wear earphones for all the experiment duration, to be isolated from external noise. The experiment started 10 minutes after tDCS onset and finished within the end of the stimulation. During the first 10 minutes of stimulation participants watched two silent cartoon videos, then they performed the Linguistic and the Control task, which order was counterbalanced within each group (for procedure details see Supplementary Materials - Section A). 

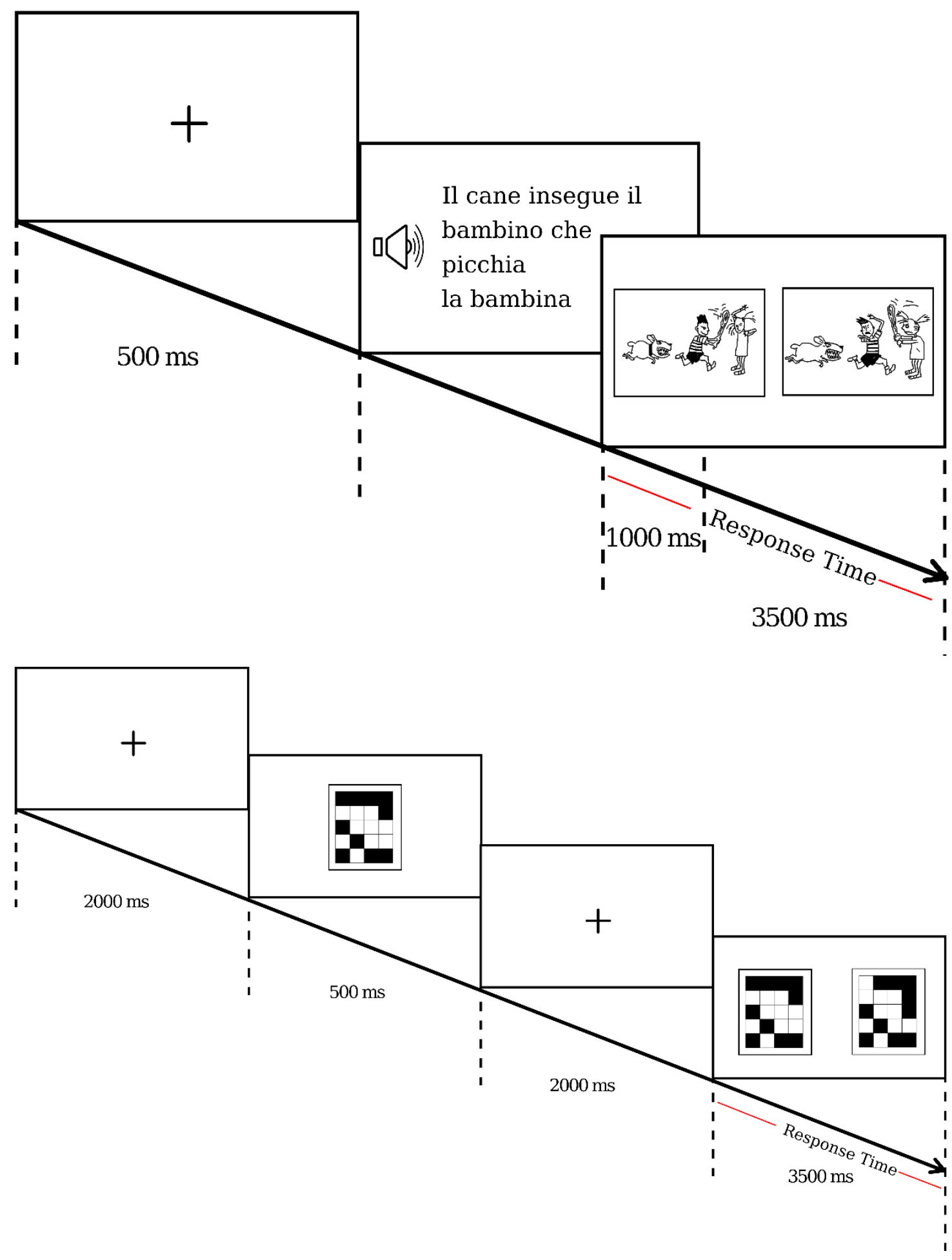

Figure 2. The figure represents a graphical scheme of our experimental procedure. Specifically, the top panel shows a trial example from our Linguistic Task, consisting in a sentence to picture matching task. In this case participants heard the sentence "Il cane insegue il bambino che picchia la bambina" (The dog is chasing the boy who is hitting the girl"). Then two pictures appeared on the screen, but only one correctly depicted the sentence meaning. Participant were required to select the right one, that in this example is the one on the left side of the screen. The timeline clarifies the overlap between the auditory stimulus and pictures presentation. The bottom panel displays an example of the Control Task, namely a visual pattern task. In this case participants saw a $5 \times 4$ size checkerboard, then have to choose among two alternatives the checkerboard equal to the first one presented. In this case the right choice was presented on the left side of the screen. 


\section{Statistical procedure}

Statistical analyses were performed in the statistical programming environment $\mathrm{R}$ ( $\mathrm{R}$ Development Core Team, 2019).

For both tasks, the dichotomous variable accuracy was analysed using general mixed effects models (Baayen et al., 2008), fitted using the GLMER function of the lme4 package (Bates et al., 2015). RTs log-transformed values were analysed according to linear mixed effects regression using the LMER procedure available in the "Ime4" R package (version 1.1-5, Bates, Maechler and Bolker, 2015) and outliers were removed via model-criticism (2.5 SD of standardized residuals, Baayen et al., 2008).

Fixed predictors inclusion in the final models have been tested with a series of likelihood ratio tests by progressively adding each parameter that significantly increased the overall model's goodness of fit (Gelman and Hill, 2006). In the linguistic task Gender (factorial, two levels: female, male), Sentence type (factorial, three levels: Sentence with coordination (Coord), relatives in center embedded position (Rel_CE) and relatives in right peripheral position (Rel_RP) and Stimulation condition (factorial, three levels: LIFG, LIPC, sham), plus their interaction were entered the model as predictors. In the control task, Stimulation condition and Gender were entered in the model as predictors.

In both tasks Participant and Item were included as random intercepts to account for participant-specific variability and for item-specific idiosyncrasies (Baayen et al., 2008). Post-hoc analyses were performed using $f d r$ correction. 


\subsection{Results}

Statistical analyses of accuracy in the linguistic task were conducted on 3414 data points ${ }^{1}$.

The best fitting model for the dependent variable accuracy was the one including the simple effects of sentence type and stimulation condition plus their interaction (see Table 1s in the Supplementary materials for mean and SE of accuracy).

The simple effect of sentence type was significant $\left(\chi^{2}(2)=8.183, p=.017\right)$ : as expected accuracy was higher for Coord as compared to both Rel_CE ( $p=.013)$ and Rel_RP ( $p=.028)$, while the two relative clauses did not differ each other $(p=.723)$.

The simple effect of stimulation condition was significant $\left(\chi^{2}(2)=43.571, p<.001\right)$, post-hoc analysis showed accuracy increasing during LIFG stimulation as compared to sham ( $\mathrm{p}=.048$ ), whereas LIPC stimulation significantly deteriorated participants' performance as compared to sham and LIFG conditions ( $\mathrm{p}<.001$ in both cases).

The interaction between stimulation condition and sentence type was also significant $\left(\chi^{2}(4)=15.005\right.$, $\mathrm{p}=.005$ ): for Coord presentation performance was better during LIFG stimulation as compared to sham $(\mathrm{p}=.012)$, while anodal tDCS over LIPC worsened accuracy as compared to sham and LIFG conditions ( $\mathrm{p}<.001$ in both cases).

For both Rel_RP and Rel_CE, LIFG stimulation did not improved performance as compared to sham ( $p=.289$ and $p=.205$, respectively), whereas tDCS over the LIPC significantly decreased accuracy as compared to sham and LIFG stimulation in both Rel_RP ( $p=.008$ and $p<.001$, respectively) and Rel_CE (p<.001 in both case, see Figure 2).

\footnotetext{
${ }^{1}$ As in the previous experiment, one sentence $\left(n^{\circ} 90\right)$ was excluded from analyses due to the low accuracy rate $(\mathbf{5 0 \%})$.
} 

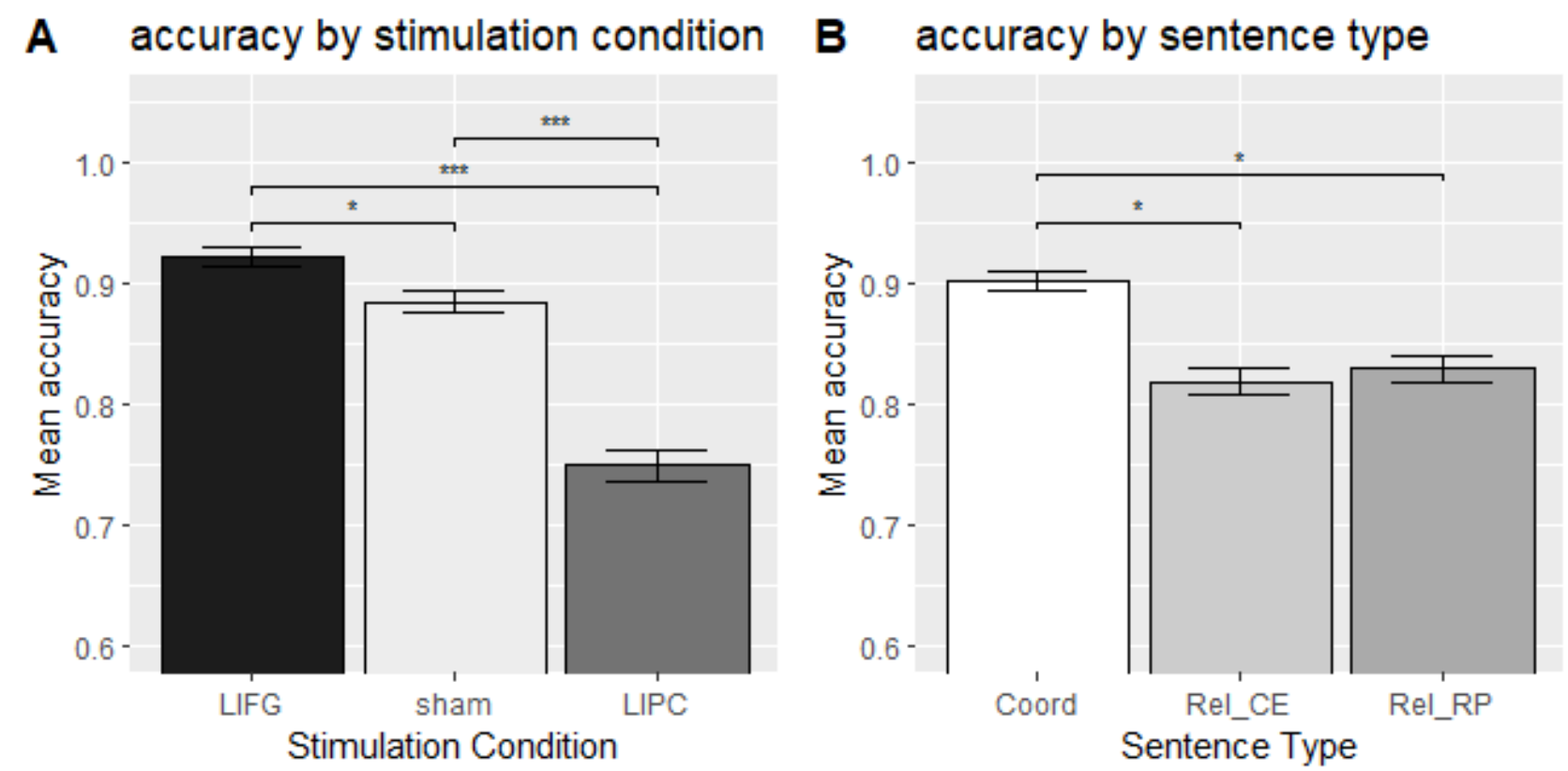

C accuracy by stimulation condition and sentence type

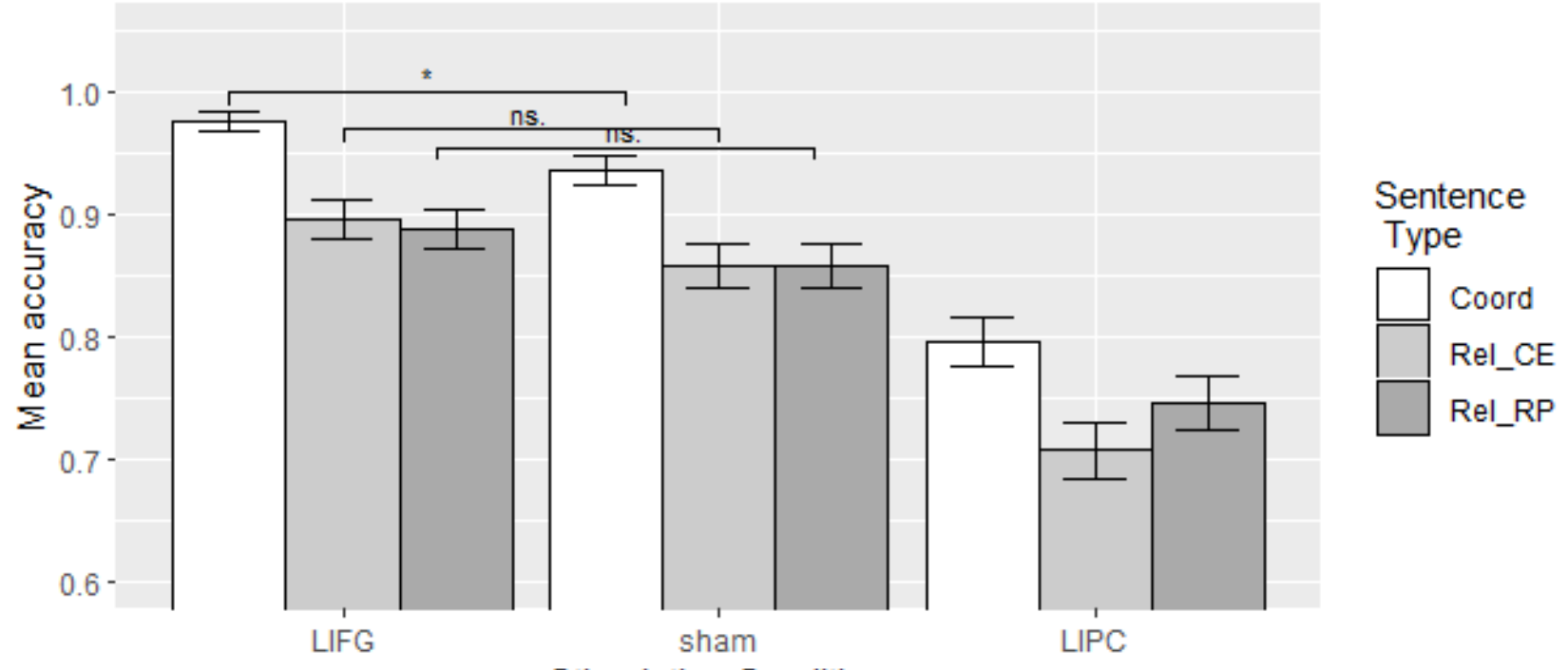

Stimulation Condition

Figure 2 represents mean accuracy (y-axis) by: A) stimulation condition; B) sentence type; and $C$ ) stimulation condition by sentence type (Coord in white, Rel_CE in light grey, Rel_RP in grey). Error bars indicate the standard error.

Panel $\mathrm{A}$ and panel $\mathrm{B}$ reports the main effects, panel $\mathrm{C}$ illustrates the interactions $(*=\mathbf{p}<.05, * * *=\mathbf{p}<.001)$, n.s. $=$ p>.05).

Concerning RTs, only accurate responses were included for analysis, which was run on 2880 data points. The best fitting model was the one with only sentence type as fixed factor (see Table $2 \mathbf{s}$ in the Supplementary materials). Participants were faster in choosing the correct picture for Coord $($ mean $=7.56, \mathrm{SE}=.029)$ as compared to Rel_RP $($ mean $=7.62, \mathrm{SE}=.029, \mathrm{p}=.021)$ and marginally 
to Rel_CE $($ mean $=7.64, \mathrm{SE}=.030, \mathrm{p}=.073)$. No difference was found between the two relative clauses $(p=.519)$.

In the VPT, accuracy was analyzed over 1180 data points and RTs, which considered accurate responses only, over $960^{2}$. As Table 1s shows for both measures the null model, including only the random intercepts, was the best fitting one, suggesting that the stimulation condition did not influence participants performance of the control task.

\section{Discussion}

The present study explored the effect of applying anodal tDCS over LIPC, a brain region considered the neural correlate of the phonological store, within the Baddeley \& Hitch's model of vSTM (Henson et al., 2000; Paulesu et al.,1993; Pisoni et al., 2019; Romero Lauro et al. 2006, 2010). The rationale behind the experiment was to complement the results of a previous study of our research group (Giustolisi et al., 2018) in which we targeted the LIFG to tap the activity of the rehearsal process, which is part of the same vSTM system of LIPC. Taken together, the two studies explored how the comprehension of sentences with different syntactic complexity is affected when the activity of LIFG and LIPC is modulated by anodal tDCS.

Interestingly, our findings showed a significant effect of the modulation induced by anodal tDCS at the behavioral level, but in two opposite directions. Indeed, as compared to the sham condition, anodal tDCS significantly enhanced participants' performance when applied over LIFG and worsened it when applied over LIPC. Crucially, no difference among the three stimulation

\footnotetext{
2 For the sake of clarity, we reported here mean values and SE of participants in the three stimulation conditions. Accuracy was the $84 \%$ (SE $=26.1$ ) and in the Sham condition, 89.7\% (SE = 27.2) during anodal tDCS over LIFG and 83.3\% (SE = 26.1) during LIPC stimulation. RTs mean were $7.02(\mathrm{SE}=.04)$ in the Sham, $7.18(\mathrm{SE}=.04)$ over LIFG, 7.21 (SE $=.042$ ) over LIPC stimulations.
} 
conditions was found in the control task, thus excluding the possibility of an a-specific effect of tDCS in our results.

Being tDCS a modulatory technique, the interference effect of the anodal stimulation over LIPC and LIFG might be interpreted as evidence for the involvement of vSTM in sentence comprehension, in line with previous evidence employing rTMS (Romero Lauro et al., 2006, 2010) and with a line of the literature on this topic (Papagno et al., 2007; Papagno \& Cecchetto 2019; Vallar \& Papagno, 2002; Vallar \& Shallice, 2007). However, we did not directly test vSTM and, furthermore, tDCS has a low spatial resolution. Therefore, it would be incautious drawing any definite specific anatomical and functional conclusions. In addition, recent neuroimaging and neuropsychological studies suggest that syntactic processing might involve not only anterior regions on the left frontal cortex but also a wider network, including temporo-parietal areas (see Friederici, 2011 for a review) among which also the inferior parietal lobule is included. Whether inferior parietal cortex plays a role in sentence comprehension by providing supporting abilities as a multiple demanding region (as suggested by Wilson et al., 2016) or in fashion more strictly related to syntactic processing (e.g. Thothathiri et al. 2012) is still unsolved. Independently from this debate, our findings support the role of LIPC in sentence comprehension and the chance to modulate it by applying tDCS on this area.

In contrast with our hypothesis, we found a decrement rather than an increment in sentence comprehension by targeting LIPC. We might speculate that increasing the activity in LIPC might in turn decrease activity in the inferior frontal gyrus due to inhibitory connections. It is known indeed that tDCS can affect other brain areas beyond the stimulated one (Holdefer et al., 2006; Miranda et al., 2006; Wagner et al., 2007) with a spread of the effect following structural and functional connections (Romero Lauro et al. 2014, 2016; Pisoni et al., 2017). Moreover, a recent mapping of the phonological loop (Papagno et al., 2017) shed light on the connecting role of the arcuate fasciculus, 
showing that the anterior segment of the AF indirect pathway, also considered as the third segment of the superior longitudinal fasciculus (SLF-III), conveys information from left BA40 to BA44.

At a more general level, our results indicate that the well-known coupling of anodal tDCSwith behavioral enhancement and cathodal with inhibition is controversial outside the sensory-motor function (Jacobson et al., 2012). Therefore, it is misleading to think that anodal tDCS over a region relevant for a task always results in a better performance in that task. It is likely that when dealing with higher cognitive functions it is necessary to assume a network perspective, in which the effect of an intervention technique over a neural hub does not linearly depend from the polarity of the stimulation but is instead the result of the complex interactions among the stimulated area and the neural regions within the network. In a clinical perspective, this latter point holds relevant implications, suggesting caution when expecting a priori polarity-dependent effect on behavior (i.e. enhancement after anodal and inhibition after cathodal), underlining instead the need to test different coupling of anodal/cathodal stimulation targeting the different hubs of the involved neural network.

Taken together, our studies confirm that sentence comprehension can be modulated by applying tDCS over LIFG and LIPC. Our previous finding (cf. Giustolisi et al., 2018) that anodal tDCS over LIFG boosts language comprehension was recently corroborated by another study (Lum et al., 2019) in which a facilitation effect on RTs was observed, targeting the same brain region and using a similar task (matching between a picture and an auditorily presented sentence). Crucially in both our first (Giustolisi et al., 2018) and present studies, tDCS effects were traceable only at the accuracy level, but did not affected RTs. We acknowledge that literature suggests that tDCS typically affects RTs more than accuracy in healthy participants (Brunoni and Vanderhasselt, 2014), but this is especially true for offline stimulation (Dedoncker et al., 2016) and can be due to task difficulty. Indeed, for tasks which are too easy at the baseline it is difficult to find any effect induced by tDCS (see for example Vergallito et al., 2018). In our sham condition, 
participants were at $86 \%$ of accuracy for the relative clause presentation, in this way we could trace both increasing and decreasing of task performance.

Differently from our and Lum et al. (2019) studies, Malyutina and colleagues (2018) did not find any modulatory effect of tDCS over LIFG. However, Malyutina and collaborators used a more lateral stimulation site, namely F7, and assessed comprehension of sentences in a different way (selfpaced reading task followed by a written question that required choosing between two possible written responses). Notably, different results might arise from different procedures, suggesting the importance of creating ad-hoc protocols, in which a specific task is coupled with specific tDCS polarity and montage. Our findings suggest as well that, although a certain imbalance between anodal and cathodal effect has been reported (Jacobson et al., 2012; Varoli et al., 2018), sentence comprehension might be boosted by applying cathodal stimulation over left BA40. Future research should verify this.

We were also interested in the effect of syntactic complexity on the modulation triggered by tDCS, and for this reason we employed relatively long sentences with different levels of syntactic complexity, ranging from coordinate clauses to more complex relative clauses in right peripheral and center embedded position. As expected, we found a main effect of sentence type, with greater accuracy and slower reaction times for sentences with coordination than for relatives, regardless of the stimulation condition. However, the interaction among the site of stimulation and type of sentence highlighted a different pattern in LIFG and LIPC stimulation. Whereas anodal tDCS over left LIPC worsened accuracy in all type of sentences regardless of syntactic complexity, in the case of LIFG the overall enhancement in sentence comprehension was primarily driven by sentences with coordination, with relatives showing only a trend toward significance in comparison to sham-baseline condition. This divergence among the different types of sentences on the overall enhancement of accuracy was not evident in our first study (Giustolisi et al., 2018) and emerged only in the conjunct statistical analysis merging the data from the two studies. The finding that the effect of anodal tDCS 
was stronger with the sentences that are syntactically less complex is consistent with the hypothesis that tDCS stimulation did not directly impact the brain areas that are responsible for syntactic processing, although our study does not allow us to clarify the precise role played by the rehearsal and by the phonological store during the comprehension of the different types of sentences.

To summarize, our studies show that it is possible to affect sentence comprehension by modulating LIFG and LIPC activity using tDCS. The result also unveils opposite effect of anodal tDCS according to the targeted site: anodal tDCS over left LFG improves sentence comprehension, whereas a disruption in performance occurred after stimulation of left LIPC. Our results are particularly interesting from a clinical perspective, paving the way to define more suitable tDCS protocols to boost standard rehabilitation treatment.

\section{References}

Baayen, R. H., Davidson, D. J., \& Bates, D. M. (2008). Mixed-effects modeling with crossed random effects for subjects and items. Journal of Memory and Language, 59, 390-412.

Baddeley, A. D., \& Hitch, G. (1974). Working memory. In Psychology of learning and motivation (Vol. 8, pp. 47-89). Academic press.

Bates, D., Maechler, M., Bolker, B., \& Walker, S. (2015). Package 'Ime4'.

Brunoni, A. R., \& Vanderhasselt, M. A. (2014). Working memory improvement with non-invasive brain stimulation of the dorsolateral prefrontal cortex: a systematic review and meta-analysis. Brain and cognition, 86, 1-9.

Butterworth, B., Campbell, R., \& Howard, D. (1986). The uses of short-term memory: A case study. The Quarterly journal of experimental psychology, 38(4), 705-737.

Caplan, D., \& Waters, G. S. (1999). Verbal working memory and sentence comprehension. Behavioral and brain Sciences, 22(1), 77-94.

Cecchetto, C., Di Domenico, A., Garraffa, M., and Papagno,C. (2012). Comprendo: batteria per la comprensione di frasi. Raffaello Cortina Editore. 
Dedoncker, J., Brunoni, A. R., Baeken, C., \& Vanderhasselt, M. A. (2016). A systematic review and meta-analysis of the effects of transcranial direct current stimulation (tDCS) over the dorsolateral prefrontal cortex in healthy and neuropsychiatric samples: influence of stimulation parameters. Brain stimulation, 9(4), 501-517.

Friederici, A. D. (2011). The brain basis of language processing: from structure to function. Physiological reviews, 91(4), 1357-1392.

Gandiga, P. C., Hummel, F. C., \& Cohen, L. G. (2006). Transcranial DC stimulation (tDCS): a tool for double-blind sham-controlled clinical studies in brain stimulation. Clinical neurophysiology, 117(4), 845-850.

Gelman, A., \& Hill, J. (2006). Data analysis using regression and multilevel/hierarchical models. Cambridge university press.

Giustolisi, B., Vergallito, A., Cecchetto, C., Varoli, E., \& Lauro, L. J. R. (2018). Anodal transcranial direct current stimulation over left inferior frontal gyrus enhances sentence comprehension. Brain and language, 176, 36-41.

Henson, R. N. A., Burgess, N., \& Frith, C. D. (2000). Recoding, storage, rehearsal and grouping in verbal short-term memory: An fMRI study. Neuropsychologia, 38(4), 426-440.

Herwig, U., Satrapi, P., \& Schönfeldt-Lecuona, C. (2003). Using the international 10-20 EEG system for positioning of transcranial magnetic stimulation. Brain topography, 16(2), 95 99.

Holdefer, R. N., Sadleir, R., \& Russell, M. J. (2006). Predicted current densities in the brain during transcranial electrical stimulation. Clinical neurophysiology, 117(6), 1388-1397.

Jacobson, L., Koslowsky, M., \& Lavidor, M. (2012). tDCS polarity effects in motor and cognitive domains: a meta-analytical review. Experimental brain research, 216(1), 1-10.

Jung, Y. J., Kim, J. H., \& Im, C. H. (2013). COMETS: A MATLAB toolbox for simulating local electric fields generated by transcranial direct current stimulation (tDCS). Biomedical engineering letters, 3(1), 39-46.

Keel, J. C., Smith, M. J., \& Wassermann, E. M. (2001). A safety-screening questionnaire for transcranial magnetic stimulation. Clinical Neurophysiology, 112(4), 720. 
Lum, J. A., Clark, G. M., Rogers, C. M., Skalkos, J. D., Fuelscher, I., Hyde, C., \& Enticott, P. G. (2019). Effects of Anodal Transcranial Direct Current Stimulation (atDCS) on Sentence Comprehension. Journal of the International Neuropsychological Society, 1-5.

Malyutina, S., Zelenkova, V., Buivolova, O., Oosterhuis, E. J., Zmanovsky, N., \& Feurra, M. (2018). Modulating the interhemispheric balance in healthy participants with transcranial direct current stimulation: No significant effects on word or sentence processing. Brain and language, 186, 60-66.

Martin, R. C., \& He, T. (2004). Semantic short-term memory and its role in sentence processing: A replication. Brain and Language, 89, 76e 82 .

Martin, R. C., \& Romani, C. (1994). Verbal working memory and sentence comprehension: A multiple components view. Neuropsychology, 8, 506e523.

Miranda, P. C., Lomarev, M., \& Hallett, M. (2006). Modeling the current distribution during transcranial direct current stimulation. Clinical neurophysiology, 117(7), 1623-1629.

Oldfield, R. C. (1971). The assessment and analysis of handedness: The Edinburgh inventory. Neuropsychologia, 9(1), 97-113.

Papagno, C., \& Cecchetto, C. (2019). Is STM involved in sentence comprehension?. Cortex, $112,80-90$.

Papagno, C., Cecchetto, C., Reati, F., \& Bello, L. (2007). Processing of syntactically complex sentences relies on verbal short-term memory: Evidence from a short-term memory patient. Cognitive Neuropsychology, 24(3), 292-311.

Papagno, C., Comi, A., Riva, M., Bizzi, A., Vernice, M., Casarotti, A., ... \& Bello, L. (2017). Mapping the brain network of the phonological loop. Human brain mapping, 38(6), 3011-3024.

Paulesu, E., Frith, C. D., \& Frackowiak, R. S. (1993). The neural correlates of the verbal component of working memory. Nature, 362(6418), 342-345.

Pisoni, A., Mattavelli, G., Casarotti, A., Comi, A., Riva, M., Bello, L., \& Papagno, C. (2019). The neural correlates of auditory-verbal short-term memory: a voxel-based lesion-symptom mapping study on 103 patients after glioma removal. Brain Structure and Function, 1-13.

Pisoni, A., Mattavelli, G., Papagno, C., Rosanova, M., Casali, A. G., \& Romero Lauro, L. (2017). Cognitive enhancement induced by anodal tDCS drives circuit-specific cortical plasticity. Cerebral Cortex (New York, NY: 1991), 1. 
Romero Lauro, L. J., Pisoni, A., Rosanova, M., Casarotto, S., Mattavelli, G., Bolognini, N., \& Vallar, G. (2016). Localizing the effects of anodal tDCS at the level of cortical sources: A Reply to Bailey et al., 2015. Cortex, 74, 323-328.

Romero Lauro, L. J., Reis, J., Cohen, L. G., Cecchetto, C., \& Papagno, C. (2010). A case for the involvement of phonological loop in sentence comprehension. Neuropsychologia, 48(14), 40034011.

Romero Lauro, L. J., Rosanova, M., Mattavelli, G., Convento, S., Pisoni, A., Opitz, A., ... Vallar, G. (2014). TDCS increases cortical excitability: Direct evidence from TMS-EEG. Cortex, 58, 99-111.

Romero Lauro, L. J., Walsh, V., \& Papagno, C. (2006). The neural correlates of phonological short-term memory: A repetitive transcranial magnetic stimulation study. Journal of Cognitive Neuroscience, 18(7), 1147-1155.

Rossi, S., Hallett, M., Rossini, P. M., \& Pascual-Leone, A., Safety of TMS Consensus Group. (2009). Safety, ethical considerations, and application guidelines for the use of transcranial magnetic stimulation in clinical practice and research. Clinical Neurophysiology, 120(12), 2008-2039.

Salis, C. (2012). Short-term memory treatment: Patterns of learning and generalisation to sentence comprehension in a person with aphasia. Neuropsychological Rehabilitation, 22(3), 428448.

Shallice, T., \& Papagno, C. (2019). Impairments of auditory-verbal short-term memory: Do selective deficits of the input phonological buffer exist?. Cortex, 112, 107-121.

Team, R. C. (2019). R: A language and environment for statistical computing. Vienna, Austria: R Foundation for Statistical Computing. https://www.R-project.org/

Thothathiri, M., Kimberg, D. Y., \& Schwartz, M. F. (2012). The neural basis of reversible sentence comprehension: evidence from voxel-based lesion symptom mapping in aphasia. Journal of cognitive neuroscience, 24(1), 212-222.

Vallar, G., \& Papagno, C. (2002). Neuropsychological impairments of verbal short-term memory. In A. D. Baddeley, M. D. Kopelman, \& B. A. Wilson (Eds.), Handbook of memory disorders (2nd ed., pp. 249-270). Chichester: Wiley.

Vallar, G., \& Shallice, T. (Eds.). (2007). Neuropsychological impairments of short-term memory. Cambridge University Press. 
Varoli, E., Pisoni, A., Mattavelli, G. C., Vergallito, A., Gallucci, A., Mauro, L. D., ... \& Lauro, L. J. R. (2018). Tracking the effect of cathodal transcranial direct current stimulation on cortical excitability and connectivity by means of TMS-EEG. Frontiers in neuroscience, 12, 319.

Vergallito, A., Lauro, L. J. R., Bonandrini, R., Zapparoli, L., Danelli, L., \& Berlingeri, M. (2018). What is difficult for you can be easy for me. Effects of increasing individual task demand on prefrontal lateralization: a tDCS study. Neuropsychologia, 109, 283-294.

Wagner, T., Fregni, F., Fecteau, S., Grodzinsky, A., Zahn, M., \& Pascual-Leone, A. (2007). Transcranial direct current stimulation: a computer-based human model study. Neuroimage, 35(3), 1113-1124.

Wilson, S. M., DeMarco, A. T., Henry, M. L., Gesierich, B., Babiak, M., Miller, B. L., \& Gorno-Tempini, M. L. (2016). Variable disruption of a syntactic processing network in primary progressive aphasia. Brain, 139(11), 2994-3006. 